Canon abweicht]. Die Berechnungen sind mit Hilfe der Schramschen ") "Tafeln zur Berechnung der năheren I!mstände der Sonnenfinsternisse für Berlin durchgeführt. Die Unsicherheit der angegebenen größten Phase beträgt wohl nur wenige Prozent. Das folgende $\mathbf{V}$ oder $\mathbf{S}$ deutet an, ob der Mondmittelpunkt dabei nördlich oder südlich vom Sonnenzentrum steht. Die letzte Spalte läßt erkennen, ob die Finsternis ringförmig oder total sein und welche Dauer sie für die Firde luberhaupt haben wird.

\begin{tabular}{|c|c|c|c|}
\hline Datusn & $\begin{array}{c}\text { Wahre } \\
\text { 7.eit Perl. }\end{array}$ & $\begin{array}{c}\text { GröBte } \\
\text { Phase }\end{array}$ & $\begin{array}{c}\text { Halbe Dauer } \\
\text { uberhaupt }\end{array}$ \\
\hline 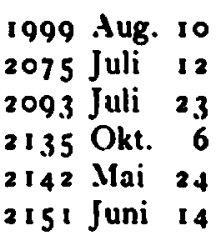 & $\begin{array}{r}23: 4 \\
17.5 \\
1.6 \\
20.5 \\
21.9 \\
7.3\end{array}$ & $\begin{array}{l}0.88 \mathrm{~S} \\
0.85 \mathrm{~S} \\
0.94 \mathrm{~S} \\
\text { total } \\
0.98 \mathrm{~N} \\
0.92 \mathrm{~S}\end{array}$ & $\begin{array}{lll}t & 2^{h} & 33^{m} \\
r & 2 & 48 \\
r & 2 & 52 \\
t & 2 & 33 \\
t & 2 & 29 \\
t & 2 & 26\end{array}$ \\
\hline
\end{tabular}

Es wird also fast zwei Jahrhunderte daucrn, bis eine Finsternis von ahnlichem Betrag wie die kommende sich bei uns ereignet.

Berlin, 19 I 2 April 2.

H. H. Áritsinger.

Tenkschr. d. Wiener Akad. d. Wiss. Bd. 51, Math.phys. KI. 1886.

\title{
Nova (18.1912) Geminorum 2.
}

Von der Nova Geminorum erhielt ich noch die folgenden Helligkeitsschätzungen (Fortsetzung zu A. N. 4563):

\begin{tabular}{|c|c|c|c|c|c|c|}
\hline \multicolumn{2}{|c|}{$\begin{array}{c}1912 \\
\text { Mărz } 14\end{array}$} & \multicolumn{2}{|c|}{ M.\%.Gr. } & I & Bemerkungen & $\begin{array}{l}\text { Größe } \\
3^{n !} \cdot 5:\end{array}$ \\
\hline & 22 & 10 & 2 I & 4 & ॥ & 4.90 \\
\hline & 23 & 7 & 18 & 2 & & 4.86 \\
\hline & 23 & 7 & 44 & 3 & Schätzung mit freiem Auge & 4 \\
\hline & 23 & 12 & 2 & 5 & & \\
\hline & 24 & I I & 3 & 1 & in Wolkenlücke & 4.6 \\
\hline & 25 & 7 & 45 & 1 & Monschein stört & 4.9 \\
\hline & 27 & 12 & 47 & 3 & 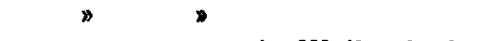 & 6.7 \\
\hline & 28 & 10 & 18 & 4 & - , in Wolkenlücke & 6.5 \\
\hline & 30 & 8 & 50 & 5 & & \\
\hline & $\mathbf{I}$ & 9 & 21 & 3 & & 6 \\
\hline & I & 9 & 24 & $\mathbf{3}$ & & 6.3 \\
\hline
\end{tabular}

Abgesehen von den Mondscheinabenden, an denen cin Handsucher benutzt wurde, sind die Schätzungen mit einem (ialileischen Doppelfernrohr ausgeführt worden. Am 23. Miärz war die Nova im Äquatorial hellgelb; im Spektrum dominierten dic Wasserstofflinien $H_{\alpha}, H_{\beta}$ und $H_{\gamma}$. Eine photographische Aufnahme mit einem Cooke-Triplet, die ich an demselben Abend I th.5 bis I I h. M. Z. Greenw. erhalten habe, zeigt die Nova nur wenig schwächer als $*$ Geminorum, etwa gleich oder $\varepsilon$ Geminorum, also nahe eine Größenklasse heller als dic visuellen Schätzungen ergeben.

Sternwarte Bergedorf, 1912 April $2 . \quad K$. Graff.

Die Nova sah ich zuerst am 14. März und schătzte sie in Wolkenlücken etwa $=\boldsymbol{*}$ Geminorum. Aufnahmen von 16. und 19. Mărz ergeben nach vorlüufiger Reduktion fir die photographische Helligkeit der Nova:

$$
\begin{aligned}
& \text { März } 169^{\text {h }} 2^{m} \text { M. E. Z. } \\
& N_{2} \text { mo5 }_{05} \text { v. } \Lambda^{r} 0^{m} 66>\mathrm{BD}+33^{\circ}{ }_{433} \\
& \text { März } 198^{\text {h }} 59^{\mathrm{m}} \text { M. E. Z. } \\
& N_{1}^{m} \cdot 79<\boldsymbol{v}, N_{0}^{m} \cdot 8_{1}>B D+33^{\circ} \text { r } 433 \text {. }
\end{aligned}
$$

Beobachtungen mit dem Okularspektroskop vom 19. 26., 29., 30. März zeigen das ganze Spektrum beherrscht von den sehr hellen und breiten Wasserstoff-Emissionslinien $H_{\alpha}, H_{i}$ und $H_{r}$ außerdem ist im Gelben eine helle Linie deutlich erkennbar, vermutlich die Heliumlinie $D_{\mathrm{y}}$; Absorptionslinien konnten bei der Lichtschwäche des kontinuierlichen Spektrums nicht mit Sicherheit festgestellt werden.

Fine Spektralaufnahme vom I9. Märi auf rotempfindlicher Platte zeigt ebenfalls die drei Wasserstofflinien $\Pi_{a}$, $H_{;}, H_{;}$init größter Deutlichkeit. Dic Iinien haben alle cine Breite von rund $30 \AA . \AA$. . .., und $z$ war ist die Seite der längeren Wellen um $9 \hat{A}$. F. nach Rot, die der kürzeren Wellen um 2 I $\AA$. E. nach Violett gegen die normale Iage der I.inien verschoben. Die I.inie $H_{y}$ besitzt ganz deutlich drei Helligkeitsmaxima bei den Wellenlängen 4348, 4339, 4332 A. F., von denen das erste das kräftigste ist, und die alle gegen Violett hin schärfer begrenzt erscheinen als gegen Rot; im ganzen ăhnelt die Firscheinung sehr den $V$ ogelschen Beobachtungen bei der Nova Aurigac. Auch in $I_{f}$ lassen sich noch Teilungen erkennen, die aber wegen der hier schon recht geringen Dispersion kaum meßbar sind, in $\boldsymbol{H}_{\alpha}$ sind sie völlig verloren gegangen; doch läßt der Umstand, daß alle drei Linien die gleiche Breite in Wellenlängen haben, wohl darauf schließen, daß ihre Struktur auch ännlich sein wird. Fine Untersuchung, ob die I.inien etwa Polarisationscrschcinungen zeigen, ergab ein negatives Resultat.

Das (jebiet zwischen $H_{f}$ und $H_{\gamma}$ zeigt eine Reihe heller und dunkler Stellen; die kräftigste Lücke liegt zwischen 4612 und $4595 \AA$. F.

Am 29. und 30. Mărz hat Herr cand. Fulda dic Nova mit Hilfe des Keilphotometers an die Stèrne $B D+34^{\circ} 1481$, $+33^{\circ} 1433,+32^{\circ} 1414$ und $+32^{\circ} 1460$ angeschlossen und findet: Mürz $29 \mathrm{roth}^{\mathrm{h}} 3 \mathrm{om}^{\mathrm{m}}$ M. E. Z. $\quad \quad V=6^{\mathrm{m}} \mathrm{m}_{3}$

$$
\text { - } 30 \text { 10 } 2 N=5.56 \text {. }
$$

Die Lichtzunahme vom 29. auf den 30 . ist auch durch gleichzeitige Helligkeitsschützungen verbürgt.

Tübingen, Sternw. Österberg, 1912 April 1. H. Rosenberg.

Inhalt zu Nr. 4566. A. A. Nijland. Beobachtungen der verinderlichen Sterne U Gerninorum, SS Aurigae und RL Pegasi. IOS. - A. A. Nijland. Beobachtungen von SS Cygni. 109. - E. Fost. Beobachtungen des Kometen $1911 \mathrm{c}$ (Brooks!. I11. - H.. Ailippoff. IHellig. keitsschitaungen des Kometen IgI I c (Brooks:. III. - H. E. Las. Saturn und sein Ring. I13. - F. Ifaym. IJer Mond. rand whithend der Sonnenfinsternis 1912 April 17. 117. - H. H. Aritzinger. Notiz zur Sonnenfinsternis am 17. April 1912. 117. - Nova (18.1912) Geminorum 2. I19. 